\title{
Update on Dantrolene in the Treatment of Anesthetic Induced Malignant Hyperthermia
}

\author{
Brandom BW*, Kang A, Sivak EL and Young MC \\ Department of Anesthesiology, Mercy Hospital UPMC, University of Pittsburgh Medical Center, USA
}

Received: December 24, 2014; Accepted: April 10, 2015, Published: April 20, 2015

*Corresponding author: Brandom BW, Department of Anesthesiology, Mercy Hospital UPMC, North American MH Registry of MHAUS, University of Pittsburgh Medical Center, Ermire Building (B) 8th floor, 1400 Locust St, Pittsburgh, PA, 15219, USA, Tel: 4122325679; E-mail: brandombw@anes. upmc.edu

\begin{abstract}
Adverse Metabolic/Muscular Reaction to Anesthesia Reports (AMRAs) received after January 1, 2007 and before December 31, 2013 in the North American Malignant Hyperthermia Registry of the Malignant Hyperthermia Association of the United States were examined with the goal of describing any changes in the administration of dantrolene, complications associated with dantrolene or with the Malignant Hyperthermia (MH) episode itself that might contribute to increased morbidity. Greater age of the patient, longer time from the beginning of the anesthetic to the first sign of $\mathrm{MH}$ and longer time from the first sign of $\mathrm{MH}$ to the administration of dantrolene were all found to be associated with increased risk of complications due to $\mathrm{MH}$. There was no significant change in the side effect profile of dantrolene from the previous report of AMRAs prior to 2007. Patients in whom Pulmonary Edema (PE) was reported received about twice as much intravenous fluid during the initial treatment of $\mathrm{MH}$ than did those in whom PE was not reported.
\end{abstract}

\section{Introduction}

Acute Malignant Hyperthermia (MH) is a rare but potentially fatal pharmacogenetic disorder that most often occurs after the administration of volatile anesthetics and/or succinylcholine $[1,2]$. Dantrolene reduces intracellular calcium in skeletal muscle through inhibition of the Ryanodine Receptor (RYR1) and possibly other mechanisms [3-5]. Dantrolene was first available for clinical use in 1979. The case-fatality rate of MH decreased from $70 \%$ in 1970 [6] to $1.4 \%$ in 2008 [7]. Review of MH cases through 2006 showed that earlier treatment with dantrolene is associated with reduced morbidity and mortality in acute $\mathrm{MH}$ episodes [8]. Since 2007 generic dantrolene has been sold in the United States by two different companies.

Anesthesia is an ever evolving field. Since the last report published describing dantrolene use [9] several major changes in anesthesia practice have occurred. Sevoflurane, desflurane, and isoflurane have largely replaced halothane. The application of regional analgesia and new intravenous drugs has allowed reduction of the concentration of any potent inhalation anesthetic. Alternatives to succinylcholine are available. After $1997 \mathrm{MH}$ was identified later during the course of general anesthesia than it had been previously [2]. Using reports of MH cases Adverse Metabolic/Muscular Reaction to Anesthesia reports (AMRAs) voluntarily submitted to the North American Malignant Hyperthermia Registry (NAMHR) Visoiu et al. observed that time to the first sign of MH differed between anesthetic agents. Anesthetics utilizing halothane and succinylcholine produced the first sign of $\mathrm{MH}$ the fastest. In the presence of isoflurane or desflurane the first sign of $\mathrm{MH}$ more often occurred in the second or third hour of the anesthetic [2].

However, the MH deaths known to the NAMHR after 2006 suggest that the death rate from $\mathrm{MH}$ due to anesthetic exposure has increased [10]. Therefore, AMRAs reporting anesthetic induced MH occurring after January 1, 2007 and before December 31, 2013, were examined. The goal of this review is to describe the complications that were reported as a result of the $\mathrm{MH}$ episode and to examine the hypothesis that the time to administration of dantrolene after the first sign of $\mathrm{MH}$ and other details of the administration of dantrolene are associated with increased morbidity following anesthetic induced MH episodes.

Complications associated with the administration of dantrolene were described in a review of AMRAs reporting MH events between 1987 and 2006 [9]. A secondary purpose of the present review is to determine if the complications associated with administration of dantrolene have changed in recent years.

\section{Methods}

The University of Pittsburgh IRB judged this study exempt, \#PR014040208. In the NAMHR 152 AMRAs reported signs of MH in events that occurred in the US or Canada between January 1, 2007 and December 31, 2013, with at least one anesthetic drug and dantrolene given at some point during the event (Figure 1).

Patient outcomes after the MH episode were documented in the AMRA reports as checkboxes. These complications included: cardiac dysfunction, change in consciousness level and/or coma, disseminated intravascular coagulation, hepatic dysfunction, pulmonary edema, and renal dysfunction. Patient survival was documented as a separate checkbox option. For this study, serious complications associated with the $\mathrm{MH}$ episode were 


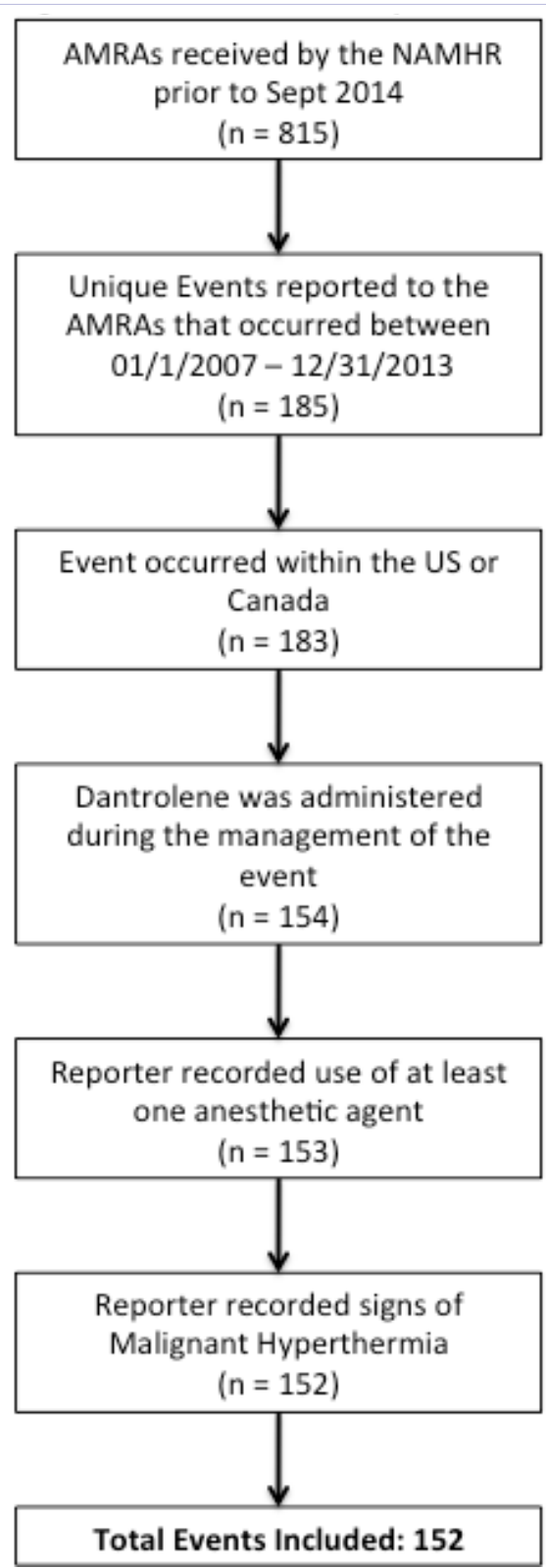

Figure 1: Flow Chart of Study inclusion.

defined as: any of the complications indicated by check boxes or described in free text or the need for defibrillation or CPR. Death during the $\mathrm{MH}$ episode or later in the same hospitalization as the $\mathrm{MH}$ episode was also considred to be a serious complication of $\mathrm{MH}$. The clinician who completes the AMRA records their opinion regarding the nature of this adverse event with a check box. The options include; adverse event not related to $\mathrm{MH}$, possible $\mathrm{MH}$, fulminant $\mathrm{MH}$ and other (with a free text field). This judgment of the reporting clinician is not determined by the Clinical Grading Scale score.

The time between the beginning of anesthetic administration and the recognition of the first sign of the $\mathrm{MH}$ episode, and the time between recognition of the first sign of $\mathrm{MH}$ and administration of the first dose of dantrolene were calculated for each case. We compared these intervals between the groups in which serious complications including death were reported, to the group without these complications.

Fluid loading, as part of the treatment of the MH episode, was documented in the AMRA with a checkbox. There was also an option to record the volume of fluid given as part of the initial treatment of the MH episode. This volume was presented as ml/ $\mathrm{kg}$.

Each $1 \mathrm{mg}$ of dantrolene in either the Dantrium or Revonto formulations of dantrolene is put into solution using $3 \mathrm{ml}$ of water. For the purpose of addressing the possible association between fluid given and complications in the subset of cases where fluid loading was noted as a treatment for the MH episode, these 2 volumes were added together.

The complications attributed to the administration of dantrolene during the $\mathrm{MH}$ episode are included in the AMRA reports as checkboxes. These complications included: phlebitis, excessive secretions, gastrointestinal upset, hyperkalemia, muscle weakness, and respiratory failure. There was also an option for the reporter to enter a free text record of other complications.

The Clinical Grading Scale (CGS) was calculated for each case, as an indicator of the severity of the MH event. The CGS has 6 categories used to assess an anesthetic induced MH episode. In each category, including rigidity, muscle necrosis, respiratory acidosis, temperature increase, cardiac involvement and other (arterial $\mathrm{pH}$ and base deficit and rapid reversal of $\mathrm{MH}$ signs after dantrolene administration), points are assigned for each observation. Cases judged to be almost certainly $\mathrm{MH}$ accumulate a total point score of 50 or more. Cases with 20 to 34 points are judged to be somewhat greater than likely to be MH [11].

SPSS 21 was used to produce descriptive statistics and perform analyses. Medians with upper and lower quartiles are presented in the text. For categorical variables, a Fisher's Exact test (FE) was used to assess the differences between the groups and the Clopper-Pearson method was used to calculate $95 \%$ confidence intervals. For continuous variables, the MannWhitney test (M-W) was used to compare the median values between groups. Logistic regression, after log transformation of time intervals, was used to describe the variables significantly associated with risk of serious complications or death associated with the MH episode. No $p$ values were corrected for multiple comparisons.

\section{Results \\ Demographics}

152 cases that occurred during the indicated time frame were examined. $66 \%$ of cases occurred in males. Median weight was $75.0 \mathrm{~kg}(48.5,97.3)$ in 149 cases where weight was recorded. Median height was $170 \mathrm{~cm}(159,178)$ in 121 cases where height was recorded. In 73 cases, $48 \%$ of the total, the reporting clinician judged that fulminant $\mathrm{MH}$ was occurring. 


\section{MH episode}

The Clinical Grading Scale (CGS), in 152 cases, ranged from 3 to 88 . The median CGS was $48(33,60)$. There was no significant correlation between CGS and time from recognition of the first sign of $\mathrm{MH}$ to administration of the first dose of dantrolene $((r=0.124 ; p=0.173$, Pearson correlation $)$. There was a closer low correlation between CGS and time from the beginning of anesthetic administration to administration of dantrolene, $(r=0.188 ; p=0.039$, Pearson correlation $)$. Thus when the patient had received anesthesia for a longer time before the administration of dantrolene, the CGS was higher.

\section{MH episode complications}

Serious complications or death associated with the $\mathrm{MH}$ episode occurred in 40 cases, constituting 26\% (20-34\%; 95\% CI) of the total cases. Death occurred in 7\% (3-12\%). Frequent serious complications associated with the $\mathrm{MH}$ episode were: cardiac dysfunction, change in level of consciousness, renal dysfunction and pulmonary edema (Table 1).

The demographics and other characteristics of these cases are presented in Table 2.

The other serious complications specifically reported with the MH episode included: compartment syndrome in three cases, pleural effusion, severe cellulitis, refractory bronchospasm, myoglobinuria, severe bleeding from all IV sites, elevated lipase with abdominal pain, and central nervous system injury, each in one case.
Table 1: Frequency of MH complications (95\% confidence intervals).

\begin{tabular}{|l|c|}
\hline Change in consciousness & $10.5 \%(6.1-16.5 \%)$ \\
\hline Cardiac Dysfunction & $11.8 \%(7.2-18.1 \%)$ \\
\hline Pulmonary Edema & $7.2 \%(3.7-12.6 \%)$ \\
\hline Renal Dysfunction & $8.6 \%(4.6-14.2 \%)$ \\
\hline DIC & $4.6 \%(1.9-9.3 \%)$ \\
\hline Hepatic Dysfunction & $5.3 \%(2.3-10.1 \%)$ \\
\hline Other & $7.2 \%(3.7-12.6 \%)$ \\
\hline
\end{tabular}

There were 112 cases with no serious complications or death reported. Both time intervals, Ind2 MH sign and Min 2 Dantrolene, were significantly longer in the group with serious complications, ( $p=0.003$ and $0.023, \mathrm{M}-\mathrm{W}$, respectively). There was also a significant difference between the group that had serious complications or death and the group that had no complications related to the MH episode in age, weight and CGS ( $p<0.001, p=$ $0.002, p=0.001$, M-W, respectively). The group with significant complications was older, weighed more and had a higher CGS (Table 2). When the initial dantrolene dose was referenced to weight $(\mathrm{mg} / \mathrm{kg}$ ) there was no difference between these groups in initial dose of dantrolene ( $\mathrm{N}=141, \mathrm{p}=0.576, \mathrm{M}-\mathrm{W})$.

\section{Most delayed administration of dantrolene}

There were 14 cases in which administration of dantrolene occurred more than 100 minutes after recognition of the first sign of MH. Plotting time from the first MH sign to administration of

Table 2: Demographic and Outcomes.

\begin{tabular}{|c|c|c|c|c|c|}
\hline \multicolumn{6}{|c|}{ Patients with serious complications or death associated with the MH episode } \\
\hline & Number of Cases & Median & $25 \%-75 \%$ & Minimum & Maximum \\
\hline Age(yr) & 39 & 41 & $30-61$ & 6 & 84 \\
\hline Weight(kg) & 38 & 86 & $65-108$ & 10 & 146 \\
\hline $1^{\text {St }}$ Dantrolene Dose(mg) & 34 & 190 & $115-253$ & 3 & 660 \\
\hline CGS & 40 & 58 & $43-63$ & 23 & 78 \\
\hline Ind2 MH Sign (min) & 37 & 126 & $60-189$ & $0^{*}$ & 660 \\
\hline Min2 Dantrolene(min) & 30 & 42 & $22-99$ & $0^{*}$ & 435 \\
\hline \multicolumn{6}{|c|}{ Patients with no complications associated with the MH episode } \\
\hline & Number of Cases & Median & $25 \%-75 \%$ & Minimum & Maximum \\
\hline Age(yr) & 109 & 24 & $9-47$ & 0 & 90 \\
\hline Weight(kg) & 111 & 70 & $32-91$ & 10 & 125 \\
\hline $1^{\text {St }}$ Dantrolene Dose(mg) & 107 & 160 & $60-220$ & 7 & 436 \\
\hline CGS & 112 & 43 & $33-53$ & 3 & 88 \\
\hline Ind2 MH Sign (min) & 102 & 63 & $26-120$ & $0^{*}$ & 600 \\
\hline Min2 Dantrolene(min) & 93 & 26 & $12-52$ & $0^{*}$ & 300 \\
\hline
\end{tabular}

Ind2 $\mathrm{MH}$ sign is the time from the beginning of anesthesia to observation of the first $\mathrm{MH}$ sign

Min2 Dantrolene is the time from observation of the first MH sign to the beginning of administration of first dose of dantrolene

CGS is calculated based on the physical sign, physiological parameters and clinic pathologic variables measured during the MH episode

Often data is missing. Therefore there is a bias in CGS to be lower than actual

*AMRA reports documented that the first sign of MH was recorded in the same minute of clock time that anesthesia was induced in six cases and that the time to administration of dantrolene was recorded in the same minute that the first MH sign was documented four cases. Seconds were not recorded. 
dantrolene showed that these cases were separated from the rest by ten minutes or more. In eight of these cases sinus tachycardia, elevated temperature, or hypercarbia was the first sign of $\mathrm{MH}$ noted. In the other six cases the first signs of MH noted included masseter muscle rigidity, generalized rigidity, hyperkalemia and decreased level of consciousness Furthermore, in nine of these 14 cases the first sign of the MH episode was not noticed until more than 100 minutes after the induction of anesthesia. In two of these cases, the first symptoms of MH did not present or were not recognized until the patient was in the intensive care unit postoperatively. In these two cases, in which positive pressure ventilation was continued after leaving the OR, dantrolene was given 137 and 240 minutes after the first sign of $\mathrm{MH}$.

Logistic regression described a significant relationship between the risk of serious complications or death and both the interval from the beginning of administration of the anesthetic to observation of the first sign of $\mathrm{MH}$, and the interval between the observation of the first sign of $\mathrm{MH}$ and the administration of dantrolene. Age of the subject was also a significant factor in this regression (Table 3 and Figure 2). For the purpose of this regression analysis, cases in which a zero time interval was reported were assigned a value of one minute. It is unlikely that dosing of dantrolene was really begun in less than one minute from the observation of the first sign of MH because dantrolene has to be reconstituted.

\section{Fluid loading}

Fluid loading was noted as part of the treatment of MH in 91 of 152 cases, $60 \%$ of the total number of reported cases. There was no significant association between risk of serious complication or death and fluid loading, when fluid loading was considered as a binary variable, ( $p=0.46, \mathrm{~F}$ E). There was no significant association between pulmonary edema and fluid loading in the entire group of 152 cases ( $p=0.20, \mathrm{~F} \mathrm{E}$ ).

The presence of fluid loading was associated with dantrolene complications in general ( $p=0.012, \mathrm{~F}$ E), in 152 cases. Of the total of 152 cases in this study, 31 cases had quantification of fluid loading. Total fluids administered during the treatment of the dantrolene episode included IV crystalloid in 31 and colloid solutions in 2 of these 31 and sterile water used to prepare the dantrolene formulation. There was no difference in age, weight, CGS, or initial dose of dantrolene between these 31 cases and the other 121 cases (Table 4).

\section{Dantrolene complications}

Complications were reported with the administration of dantrolene in 61 of 152 cases. In those cases in which complications were reported the median age was 39 years in 60 cases $(18,62)$ and weight $76(55,95) \mathrm{kg}$. The median CGS was 51 $(33,59)$ in these reports. Those patients with complications were older $(p=0.016, \mathrm{M}-\mathrm{W})$, but there was no significant difference in weight, initial dantrolene dose $(\mathrm{mg} / \mathrm{kg})$, or CGS between those cases with or without complications reported with dantrolene ( $p=0.601,0.976$ and $0.369, \mathrm{M}-\mathrm{W}$, respectively). The most frequently reported complications were muscle weakness and phlebitis. Other reported complications of dantrolene, in order of
Table 3: Logistic Regression for odds of serious complication or death.

\begin{tabular}{|l|c|c|c|}
\hline \multicolumn{1}{|c|}{ Variable } & Parameter & Standard error & Estimate \\
\hline Age & 0.041 & 0.012 & 0.001 \\
\hline LnMin2D & 0.691 & 0.241 & 0.004 \\
\hline LnInd2sign & 0.618 & 0.233 & 0.008 \\
\hline Constant & -7.824 & 1.693 & 0.000 \\
\hline
\end{tabular}

Age is the age in years at the time of MH episode

LnM2D is the natural log of time from observation of the first MH sign to administration of dantrolene

LnInd2sign natural log of time from beginning of anesthesia to observation of first MH sign

p associate with this model is $<0.00001$, including the constant

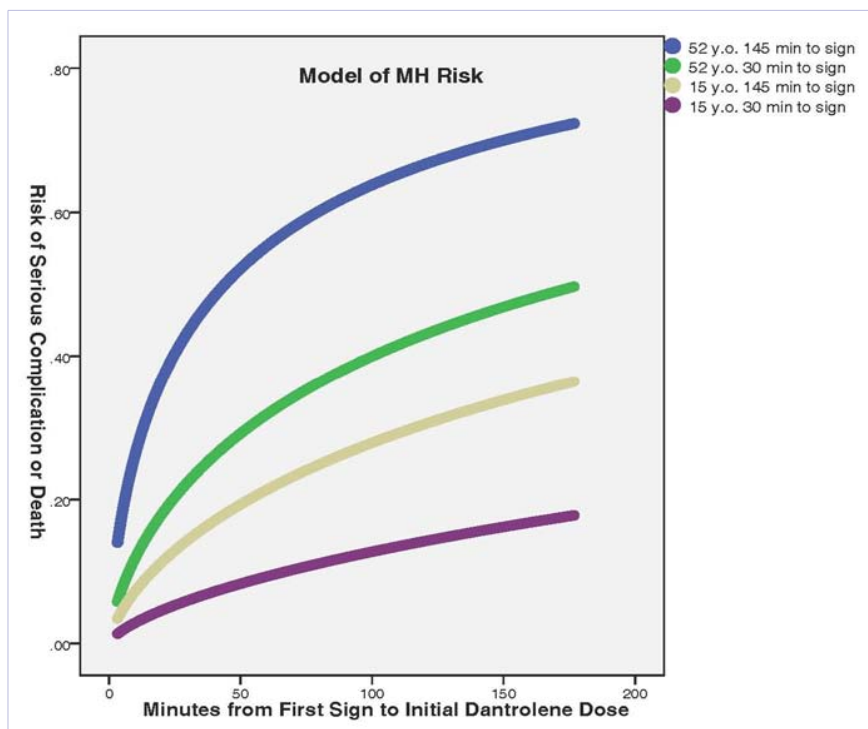

Figure 2: Model of MH Risk. The ages and time selected for the graph are values at the upper and lower quartile ranges for this data.

decreasing frequency were: hyperkalemia, respiratory failure, GI upset and excessive secretions (Table 5)

The other complications specifically listed as associated with dantrolene included: hyponatremia in 3 cases, ventilation required for $>24$ hours in 2 , nausea in 2 , and in one case each, unable to give full dose due to hypotension, unable to give full dose of dantrolene due to difficulty in mixing, two RNs needed to mix dantrolene, generalized myalgia, mild pulmonary edema, elevated liver enzymes and total bilirubin, diplopia, cellulitis, and pain without inflammation at the IV site.

\section{Discussion}

Previous analyses of similar data from the NAMHR found that longer anesthetic exposures before dantrolene was administered were associated with higher peak temperatures [10]. That study examined AMRAs until the end of 2012 and addressed the likelihood of dying from $\mathrm{MH}$, but did not examine the interval of time from the first sign of MH to the administration of dantrolene for association with risk of serious complications from the $\mathrm{MH}$ episode. The AMRAs were examined again for this study. An earlier report by Larach examining data from the NAMHR prior to 2007 noted the likelihood of any complication associated with 
Table 4: Characteristics of 31 cases with quantified fluid loading.

\begin{tabular}{|l|c|c|c|c|}
\hline & Median & $\mathbf{2 5 \% - 7 5 \%}$ & Minimum & Maximum \\
\hline Age(yr) & 36 & $12-15$ & 1 & 78 \\
\hline Weigth(kg) & 78 & $49-90$ & 10 & 125 \\
\hline 1 $^{\text {st }}$ Dantrolene Dose(mg) & 180 & $100-260$ & 3 & 360 \\
\hline CGS & 48 & $33-60$ & 15 & 78 \\
\hline Fluid Load(ml/kg) & 37 & $25-52$ & 15 & 88 \\
\hline
\end{tabular}

Table 5: Frequency of Dantrolene Complication (95\% confidence intervals).

\begin{tabular}{|l|c|}
\hline Muscle Weakness & $20.4 \%(14.3-27.7 \%)$ \\
\hline Phlebitis & $9.9 \%(5.6-15.8 \%)$ \\
\hline Hyperkalemia & $6.6 \%(3.2-11.8 \%)$ \\
\hline Respiratory Failure & $2.6 \%(0.7-6.6 \%)$ \\
\hline GI Upset & $2.0 \%(0.4-4.7 \%)$ \\
\hline Other & $12.5 \%(7.7-18.8 \%)$ \\
\hline
\end{tabular}

the MH episode increased 1.61 times for every 30 minute increase in time between the first sign of $\mathrm{MH}$ and the first administration of dantrolene [8]. The model presented in the present report specifies that the odds of a serious complication or death increase; by $49 \%$ when patient age increases by 10 years, by $53 \%$ when the time from induction of anesthesia to the first sign of $\mathrm{MH}$ doubles, and by $61 \%$ when the time from the first sign of MH to administration of dantrolene doubles. The present model predicts that the risk of complications increases 2.27 times when the time from the first sign of $\mathrm{MH}$ to administration of dantrolene increases from $10 \mathrm{~min}$ to $40 \mathrm{~min}$, using median age of $29.5 \mathrm{yrs}$ and median time from induction of anesthesia to the first sign of $\mathrm{MH}$ of $87 \mathrm{~min}$.

The current report goes further than previous studies to identify separately the increase in risk of complications associated with length of anesthesia prior to the first sign of $\mathrm{MH}$ and the interval between recognition of the first suspected $\mathrm{MH}$ sign and administration of dantrolene.

The anesthesia provider cannot alter the age and weight of the patient. Alterations in anesthesia practice that might reduce time between the beginning of anesthetic administration and recognition of the first sign of $\mathrm{MH}$ have not been formally examined, with the exception that recent data demonstrates that core temperature monitoring [10] offers the best opportunity of identifying $\mathrm{MH}$ before it becomes lethal. When monitoring is optimized and $\mathrm{MH}$ is identified early, rapid administration of dantrolene can reduce the risk of complications further.

In fourteen cases, delay from the first sign of $\mathrm{MH}$ to administration of dantrolene was greater than 100 minutes (See cases listed in Appendix) In some cases, changes in vital signs were recognized, but MH was not diagnosed quickly thereafter because of the subtle and nonspecific first signs of MH. Thus, there may have been a lower clinical suspicion of $\mathrm{MH}$ at the beginning of these MH episodes. The presentation of acute anesthetic induced MH is variable $[1,2,8]$. In the Intensive
Care Unit changes in hemodynamic stability of a patient may be attributed to post operative stress, emergence from anesthesia, underlying life-threatening conditions or factors other than $\mathrm{MH}$. While early acute $\mathrm{MH}$ reactions tend to attract attention, more delayed MH reactions may raise less suspicion.

This report supports the conclusion that dantrolene should be administered as soon as possible after the recognition of signs of $\mathrm{MH}$ due to the potential for serious complications or fatalities with delayed treatment. In addition, the incidence of complications and likely costs of treatment suggest that earlier administration of dantrolene may decrease the overall cost of hospitalization. As discussed, as the time to dantrolene administration increases, there is a greater increase in the chance of serious complications associated with the MH episode, including cardiac dysfunction, renal dysfunction, DIC, change in level of consciousness, and pulmonary edema. While most surgical procedures do not require post-operative ICU monitoring, the serious complications associated with $\mathrm{MH}$ often require a longer recovery time and admission to ICU for days. With ICU costs contributing to a third of hospital costs, admissions to the ICU have a large impact on the costs of healthcare. ${ }^{11}$ Even short term ICU monitoring has large implications for the cost of a hospital stay. In a 2005 study, daily costs in ICU units were found to be greatest on the first day of admission (\$6667 without mechanical ventilation) and stabilized by day three ( $\$ 3496$ without mechanical ventilation) [12]. Thus, by decreasing the need for extended ICU care, there is likely a large benefit in cost reduction.

Our data found no significant difference in the frequency of complications reported with administration of dantrolene from 2007 to 2013, than was reported previously [9]. The incidence of each previously reported complication [9] is within the $95 \% \mathrm{CI}$ found in this study. It is noteworthy that after 2007 dantrolene was produced by two companies that make generic drugs. Data in the NAMHR supports the view that there is no significant difference between the earlier formulation of dantrolene and that made by the first two producers of generic dantrolene. Reconstitution of dantrolene prior to 2014 required injection of $60 \mathrm{ml}$ of sterile water to produce $20 \mathrm{mg}$ of dantrolene for injection. Currently there is a new formulation of dantrolene which requires only $5 \mathrm{ml}$ of sterile water to reconstitute $250 \mathrm{mg}$ of dantrolene. Reduction in the mandatory volume of fluid needed, from $7.5 \mathrm{ml} /$ $\mathrm{kg}$ to $0.05 \mathrm{ml} / \mathrm{kg}$ to deliver $2.5 \mathrm{mg} / \mathrm{kg}$ of dantrolene may reduce the incidence of pulmonary edema associated with the treatment of MH. As this new form of dantrolene is introduced into clinical practice it will be important to collect data to compare the formulations.

We express our sympathy to the families of those patients who suffered MH episodes. We thank the many anesthesiologists and other health care providers who submitted AMRAs to the NAMHR and the Malignant Hyperthermia Association of the United States and the Department of Anesthesiology in the University of Pittsburgh for their support of the NAMHR over many years. 


\section{References}

1. RiaziS, Larach MG, Hu C, Wijeysundera D, Massey C, Kraeva N. Malignant hyperthermia in Canada: Characteristics of index anesthetics in 129 malignant hyperthermia susceptible probands. Anesth Analg. 2014; 118(2): 381-7. doi: 10.1213/ANE.0b013e3182937d8b.

2. Visoiu M, Young MC, Wieland K, Brandom BW. Anesthetic drugs and onset of malignant hyperthermia. Anesth Analg. 2014; 118(2): 38896. doi: 10.1213/ANE.0000000000000062.

3. Zhao F, Li P, Chen SRW, Louis CF, Fruen BR. Dantrolene Inhibition of Ryanodine Receptor Ca2+ Release Channels Molecular Mechanism and Isoform Selectivity. J Biol Chem. 2001; 276(17): 13810-16.

4. Zhao X, Weisleder N, Han X, Pan Z, Parness J, Brotto M, et al. Azumolene inhibits a component of store-operated calcium entry coupled to the skeletal muscle ryanodine receptor. J Biol Chem. 2006; 281(144): 33477-86.

5. Yarotskyy V, Protasi F, Dirksen RT. Accelerated activation of SOCE current in myotubes from two mouse models of anesthetic- and heatinduced sudden death. PLoS One. 2013; 8(10): e77633. doi: 10.1371/ journal.pone.0077633.

6. Denborough M. Malignant hyperthermia. Lancet. 1998; 352(9134): 1131-6.

7. Larach MG, Brandom BW, Allen GC, Gronert GA, Lehman EB. Cardiac arrests and deaths associated with malignant hyperthermia in North
America from 1987 to 2006: a report from the North American Malignant Hyperthermia Registry of the Malignant Hyperthermia Association of the United States. Anesthesiology. 2008; 108(4): 60311. doi: 10.1097/ALN.0b013e318167aee2.

8. Larach MG, Gronert GA, Allen GC, Brandom BW, Lehman EB. Clinical presentation, treatment, and complications of malignant hyperthermia in North America from 1987 to 2006. Anesth Analg. 110(2): 498-507. doi: 10.1213/ANE.0b013e3181c6b9b2.

9. Brandom BW, Larach MG, Chen MS, Young MC. Complications associated with the administration of dantrolene 1987 to 2006: a report from the North American Malignant Hyperthermia Registry of the Malignant Hyperthermia Association of the United States. Anesth Analg. 2011; 112(5): 1115-23. doi: 10.1213/ANE.0b013e31820b5f1f.

10. Larach MG, Brandom BW, Allen GC, Gronert GA, Lehman EB. Malignant hyperthermia deaths related to inadequate temperature monitoring, 2007-2012: a report from the North American Malignant Hyperthermia Registry of the Malignant Hyperthermia Association of the United States. Anesth Analg. 2014; 119(6): 1359-66. doi: 10.1213/ ANE.0000000000000421.

11. Kahn JM. Understanding economic outcomes in critical care. Curr Opin Crit Care. 2006; 12(5): 399-404.

12. Dasta, JF, McLaughlin TP, Mody, SH, Piech, CT. Daily costs of an intensive care unit day: The contribution of mechanical ventilation. Crit Care Med. 2005; 33(6): 1266-71. 